\title{
Momentum Theory with Slipstream Rotation Applied to Wind Turbines
}

\author{
D. F. Hunsaker ${ }^{*}$ and W. F. Phillips ${ }^{\dagger}$ \\ Utah State University, Logan, Utah 84322-4130
}

\begin{abstract}
A momentum theory which includes the effects of slipstream rotation for wind turbines is presented. The theory accounts for the axial and radial pressure gradients within the slipstream as well as the wake expansion caused by wake rotation. Because of the limiting approximations of previous methods, the effects of slipstream rotation have not been accurately realized. The method included here, which does not suffer from the unrealistic approximations of previous methods, predicts that the effects of slipstream rotation are manifest entirely through an increase in the turbine thrust coefficient. The method predicts, as previous methods do, that the Lanchester-Betz-Joukowski limit of 16/27 is an upper limit for the maximum efficiency, or power coefficient, of a wind turbine. Unlike the results from classical methods that are traditionally reported in terms of the axial induction factor, results of this work are presented in terms of two independent variables, the tip-speed ratio and the torque coefficient. The results included here allow the dependent variables including the thrust coefficient, power coefficient, axial induction factor, and circumferential induction factor to be evaluated in terms of the tip-speed ratio and torque coefficient. Additionally, relationships for the ideal operating conditions of a wind turbine are presented.
\end{abstract}

\section{Nomenclature}

$=$ variable defined in Eq. (66)

$=$ cross-sectional area of the ultimate slipstream

$=$ area of the turbine disk

$=$ axial induction factor, $a \equiv V_{x i} / V_{\infty}$

$=$ circumferential induction factor, $a^{\prime} \equiv V_{\theta p} /\left(\omega_{t} r_{t}\right)$

$=$ variable defined in Eq. (66)

$=$ power coefficient defined in Eq. (13)

$=$ torque coefficient defined in Eq. (58)

$=$ thrust coefficient defined in Eq. (12)

$=$ ultimate slipstream enthalpy

$=$ freestream enthalpy

$=$ mass flow through the turbine disk

$=$ power extracted from the fluid by the turbine

$=$ pressure

$=$ pressure immediately downstream of the turbine disk

$=$ pressure in the ultimate slipstream

$=$ pressure immediately upstream of the turbine disk

$=$ freestream pressure

$=$ torque produced by the fluid on the turbine

$\begin{array}{ll}R_{s} & =\text { radius of the ultimate slipstream } \\ R_{t} & =\text { radius of the turbine disk }\end{array}$

\footnotetext{
* PhD Candidate, Mechanical and Aerospace Engineering Department, 4130 Old Main Hill; currently Design Engineer, Scaled Composites, 1624 Flight Line, Mojave, CA 93501-1663. Member AIAA.

${ }^{\dagger}$ Professor, Mechanical and Aerospace Engineering Department, 4130 Old Main Hill. Senior Member AIAA.
} 


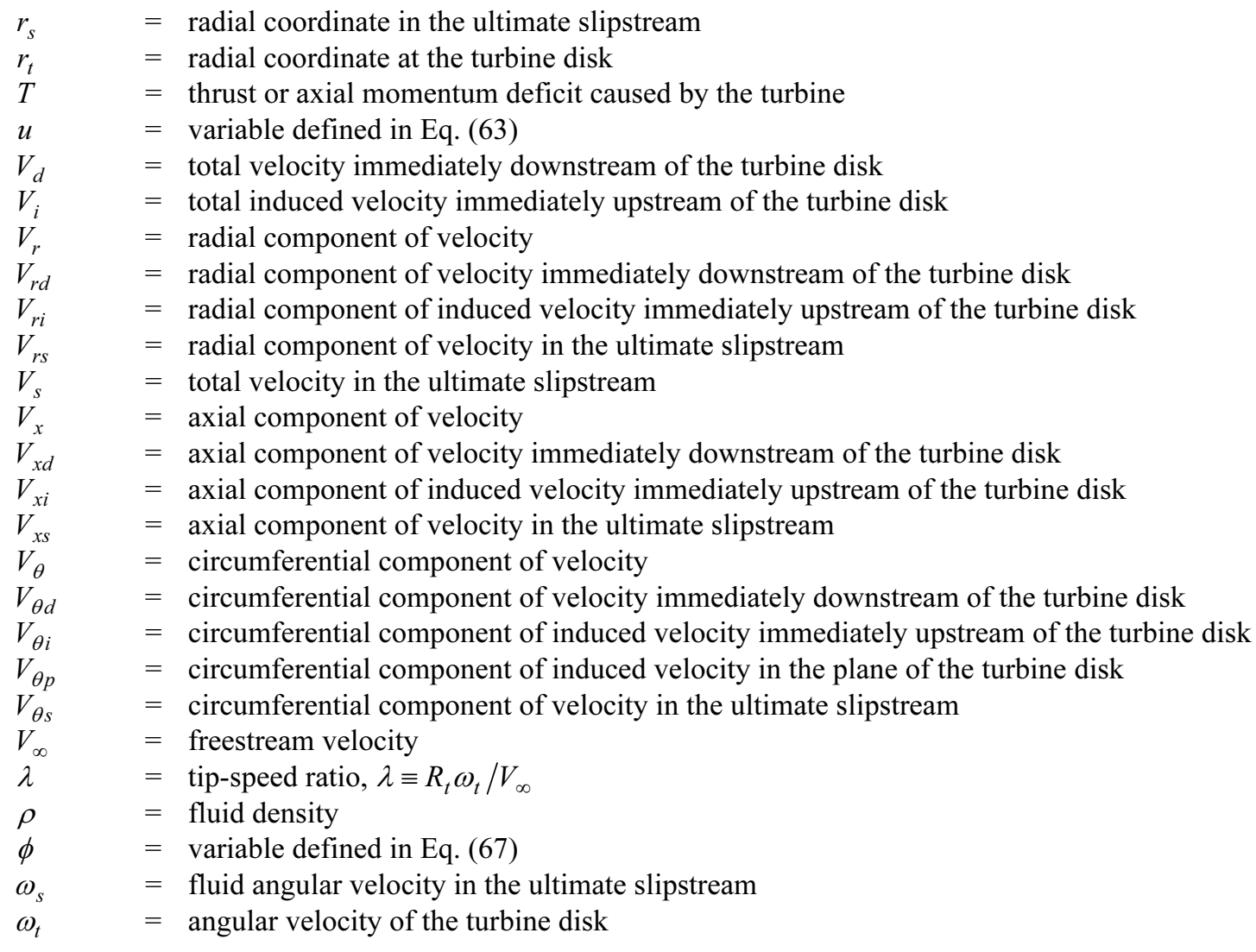

\section{Introduction}

The ideal efficiency of a wind turbine is often referred to as the Betz limit named after the German scientist Albert Betz who published his findings on the theory in $1920 .{ }^{1}$ However, two other researchers published very similar results about the same time as Betz. Frederick Lanchester, an English scientist, was the first to publish the result in $1915 .^{2}$ It is unclear whether or not Betz was aware of the development of Lanchester and therefore it has been suggested that the limit be referred to as the Lanchester-Betz limit. ${ }^{3}$ In 1920, the Russian scientist N. E. Joukowski (Nikolai Zhukovsky) also developed the theoretical limit. ${ }^{4}$ According to Kuik, ${ }^{5}$ there seems to be no reason to assume that Joukowski was aware of the development of Lanchester. Because it is possible that each of these scientists developed this theoretical limit independent of the others, it has been suggested that the ideal efficiency be referred to as the Lanchester-Betz-Joukowski limit in scientific writing. ${ }^{5}$

The original momentum and blade element theories used to predict the efficiency of any system of rotating blades were based on the development of propellers, and are thus often termed propeller momentum or propeller blade-element theories. However, the theories are well known to apply to the reverse problem of a wind turbine where power is extracted from the flow rather than imparted to the flow. ${ }^{6}$ A critical component of any propeller theory is the prediction of the axial component of induced velocity on the turbine disk as a result of lift developed by the blades. This is termed the axial induction factor and is defined as the ratio of the axial component of induced velocity at the turbine disk to the freestream velocity. In most momentum theories, the axial induction factor is denoted by the symbol $a$ and is generally treated as an independent variable. The power coefficient of a wind turbine is commonly referred to as the efficiency of the wind turbine and is defined as the ratio of the power extracted by the turbine from the fluid to the power available in the freestream integrated over the area of the turbine disk, $C_{P} \equiv P /\left(\frac{1}{2} \rho V_{\infty}^{3} A_{t}\right)$. Classical momentum theory predicts that the optimum operating condition exists at an axial induction factor of $1 / 3$ which corresponds to a maximum power coefficient of 16/27 and a thrust coefficient of 8/9.

Interest in the theoretical ideal efficiency of wind turbines has escalated recently as evidenced by the increased number of publications on the subject. This interest is likely due to the heightened awareness of the need for clean energy and has renewed studies in the development of wind and water turbines. The original developments of the classical momentum theories were published in the early 1900s, and recent work has endeavored to increase the 
fidelity or expand the application of the original models. For example, Gorban, Gorlov, and Silantyev ${ }^{7}$ use a type of conformal mapping to obtain an improved approximation for the flowfield from which the efficiency can be obtained. They conclude that the maximum efficiency of a conventional horizontal axis turbine is $30.1 \%$, and that of a vertical axis turbine is $35 \%$. Cuerva and Sanz-Andrés ${ }^{8}$ suggest that the optimum axial induction factor varies slightly from $1 / 3$ when operational conditions such as the cost of energy and the degradation of the turbine are taken into account. Jamieson ${ }^{9}$ extends momentum theory to account for the influence of diffusers, ducts, or other objects that affect the flow and concludes that the ideal operating condition is always at a thrust coefficient of 8/9 regardless of the surrounding objects. Okulov and Sørensen ${ }^{10}$ build on the work of Goldstein ${ }^{11}$ to develop a model that is capable of predicting the theoretical limit for a turbine with a finite number of blades and claim that it is consistent with models based on momentum theory. Huleihil ${ }^{12}$ suggests that the ideal efficiency of a turbine should be closer to $47.7 \%$ based on an analogy with heat engines. Werle ${ }^{13}$ applies a form of classical momentum theory to evaluate blockage effects that can be used to correct performance parameters measured on wind turbines in wind tunnels.

Models such as those discussed above have been very helpful for designing and operating propellers and wind turbines for many years. However, many of the models in use today are based on the original momentum theory which tacitly neglects slipstream rotation. There appears to be no physical basis for neglecting slipstream rotation. Clearly, the turbine must extract power from the flow through the torque applied to the turbine, and that torque must result in rotation of the fluid within the slipstream. Those models which do attempt to include the effects of slipstream rotation are commonly based on the original work of Joukowski ${ }^{14}$ and Glauert ${ }^{15}$ which suffers from unrealistic approximations for the rotating wake. A brief discussion on this classical work is insightful.

Joukowski, ${ }^{14}$ and later Glauert, ${ }^{15}$ included the effects of wake rotation in a momentum theory model for propellers. However, in order to obtain a closed-form solution for the propeller torque, it was assumed that the flow in the slipstream is irrotational except along the axis. This assumption led to the approximation that the rotational momentum per unit mass, $\omega r^{2}$, in the slipstream is independent of radius. Such an approximation results in a model which predicts that the circumferential velocity in the slipstream is inversely proportional to radius, as would be the case if a single potential vortex filament were placed along the axis of the propeller in the slipstream. For such a model, rotational velocities near the centerline of the slipstream exceed the rotational velocity of the turbine, and the rotational velocity at the slipstream centerline becomes infinite. This is clearly an unrealistic model for the flowfield behind a propeller or a wind turbine. Alternatively, Glauert suggests an approximate solution which is obtained by assuming that the angular velocity imparted to the wake is small compared to the angular velocity of the turbine disk. While this may be true, application of this assumption led to the approximation that the pressure in the fully developed wake is uniform and equal to the atmospheric pressure. Again, this is clearly an unrealistic approximation for the flowfield behind a propeller or wind turbine. The rotation in the wake requires a pressure gradient to balance the centrifugal force on a rotating mass of fluid, causing a decrease in pressure with decreasing radius. Application of either of these two classical approximations results in a model not fully capable of correctly accounting for the physics of wake rotation.

Although some have pointed out the unrealistic nature of these approximations, subsequent models attempting to account for slipstream rotation have often been based on applying one of these historical approximations. Sharpe ${ }^{16}$ points out that the pressure in the fully developed wake is not uniform and not equal to the atmospheric pressure because the rotational momentum of the fluid in the wake must be balanced by a radial pressure gradient. The inconsistency of ignoring the pressure gradient in the rotating wake is also discussed by de Vries. ${ }^{17}$ Recent work based on approximations similar to those of Glauert include the work of Wilson and Lissaman ${ }^{18}$ and Sharpe ${ }^{16}$ From a physical standpoint, if a momentum model for propeller or wind turbine flow is desired for correctly predicting the effects of wake rotation, it must be based on assumptions that match the physics of the flow.

This paper presents a momentum theory capable of accounting for both the translational and rotational momentum of the fluid within the slipstream of the turbine and does not suffer from the unrealistic approximations discussed above. The model is capable of accounting for both the pressure gradient and rotational momentum associated with slipstream rotation. The work presented here is based on a propeller momentum theory model developed by Phillips. ${ }^{19}$ Application of this model to the problem of a wind turbine provides insight into the effects of wake rotation on the ideal operating conditions of a turbine.

Although results of previous momentum theories are often reported in terms of the axial induction factor, the results of this model are reported in terms of the tip-speed ratio and torque coefficient. This is significant because the tip-speed ratio and torque coefficient are independent variables while the axial induction factor is a dependent variable. Examples of other dependent variables of a wind turbine include the power and thrust coefficients. 
Therefore, an additional contribution of this work is the development of the axial induction factor as a function of the tip-speed ratio and torque coefficient.

This paper includes a detailed version of the classical momentum theory followed by the development of a momentum theory that includes the effects of slipstream rotation. Although the classical momentum theory is well established in the literature, it is included here so that the reader can see the direct analogy between the classical momentum theory and the momentum theory presented as the topic of this paper.

\section{Classical Momentum Theory}

Classical momentum theory can be found in several books on wind energy and aeronautical engineering. ${ }^{20-24}$ Momentum theory is based on the hypothesis of a streamtube, which encloses the complete turbine disk as shown in Fig. 1. This tube is assumed to extend infinitely far upstream from the turbine disk to a plane infinitely far downstream. All of the fluid that enters this streamtube on the far upstream side must pass through the turbine disk and exit the streamtube on the far downstream side.

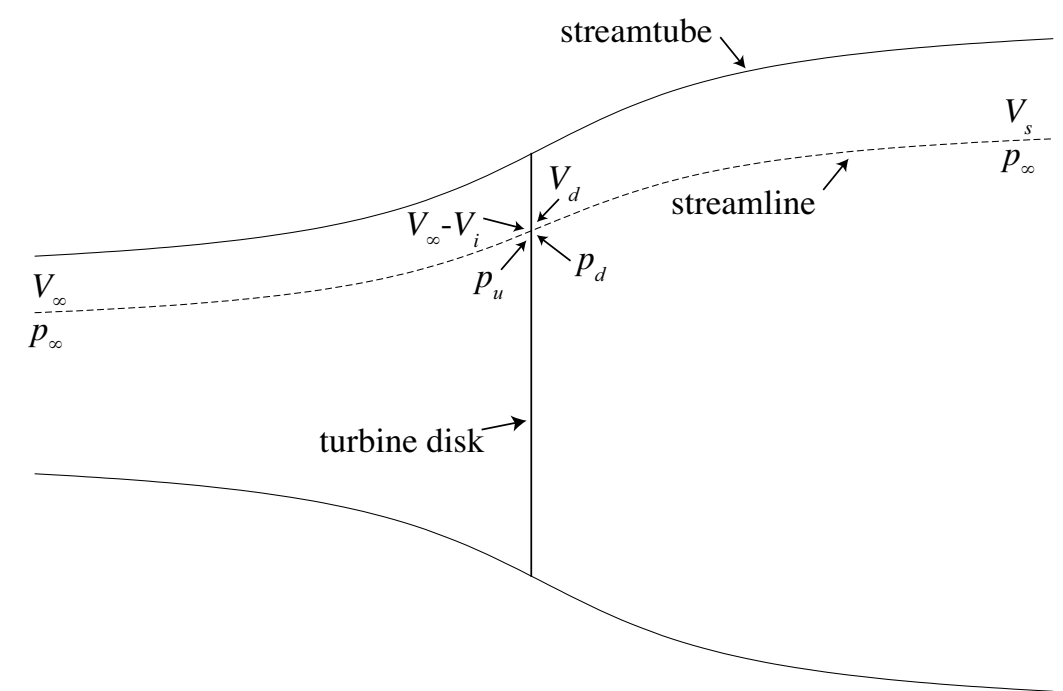

Figure 1. Classical momentum theory model for the flow about a turbine disk.

In addition to the foundational hypothesis of streamtube flow shown in Fig. 1, classical momentum theory imposes five simplifying approximations. The flow is assumed to be 1) inviscid and 2) incompressible; 3) all rotation of the fluid within the streamtube is neglected; and both 4) the velocity and 5) the static pressure are assumed uniform over each cross section of the streamtube.

To evaluate the induced velocity from classical momentum theory, we consider the flow along the streamline shown in Fig. 1. The fluid far upstream from the turbine disk has pressure $p_{\infty}$ and velocity $V_{\infty}$. As a result of the momentum deficit caused by the turbine, the pressure just upstream from the turbine disk is above ambient, while the pressure just downstream from the turbine disk is below ambient. The near upstream and downstream pressures are denoted as $p_{u}$ and $p_{d}$, respectively. By definition, the velocity on the near upstream side of the turbine disk is the freestream velocity minus the induced velocity, $V_{\infty}-V_{i}$, and the velocity on the near downstream side is designated as $V_{d}$. The radial component of velocity at all streamtube cross-sections is neglected in classical momentum theory.

Because no energy is extracted or added to the flow upstream of the turbine disk, Bernoulli's equation can be applied to relate the velocities and pressures in the freestream to those at the upstream side of the turbine disk

$$
\frac{p_{\infty}}{\rho}+\frac{V_{\infty}^{2}}{2}=\frac{p_{u}}{\rho}+\frac{\left(V_{\infty}-V_{i}\right)^{2}}{2}
$$

Likewise, Bernoulli's equation can be used to relate the pressure and velocity on the immediate downstream side of the turbine disk to those in the slipstream because no energy is added or extracted from the flow in this region 


$$
\frac{p_{\infty}}{\rho}+\frac{V_{s}^{2}}{2}=\frac{p_{d}}{\rho}+\frac{V_{d}^{2}}{2}
$$

Based on the assumption of incompressible flow, conservation of mass just upstream and downstream of the turbine disk requires that

$$
V_{d}=V_{\infty}-V_{i}
$$

Using Eq. (3) in Eq. (2) and subtracting Eq. (2) from Eq. (1) gives

$$
\frac{V_{\infty}^{2}}{2}-\frac{V_{s}^{2}}{2}=\frac{p_{u}}{\rho}-\frac{p_{d}}{\rho}
$$

The axial momentum deficit of the fluid results in an axial force commonly termed the thrust of the turbine. The fluid exerts this force on the turbine in the direction of the freestream velocity, and as a result, the turbine must produce a reacting force in the opposite direction. The thrust can be written in terms of the pressure difference on the upstream and downstream side of the turbine disk, or in terms of the change in axial velocities far upstream and downstream of the turbine disk

$$
T=A_{t}\left(p_{u}-p_{d}\right)=\dot{m}\left(V_{\infty}-V_{s}\right)
$$

where the mass flux $\dot{m}$ can be written as the mass flow through the turbine disk

$$
\dot{m}=\rho A_{t}\left(V_{\infty}-V_{i}\right)
$$

Combining Eqs. (4), (5), and (6) gives an expression for the slipstream velocity in terms of the freestream velocity and induced velocity

$$
V_{s}=V_{\infty}-2 V_{i}
$$

Using Eqs. (7) and (6) in Eq. (5), the expression for thrust can be written as

$$
T=2 \rho A_{t} V_{i}\left(V_{\infty}-V_{i}\right)
$$

From the first law of thermodynamics, the power extracted from the fluid by the turbine must satisfy the relation

$$
P=\dot{m}\left(h_{\infty}-h_{s}+\frac{V_{\infty}^{2}}{2}-\frac{V_{s}^{2}}{2}\right)
$$

where $h_{\infty}$ and $h_{s}$ are, respectively, the freestream and ultimate slipstream enthalpy. Because the fluid is assumed incompressible and the pressure in the ultimate slipstream is assumed to be the same as in the freestream, the enthalpy in the ultimate slipstream is equal to the enthalpy in the freestream. Using Eqs. (7) and (6) in Eq. (9) gives

$$
P=2 \rho A_{t} V_{i}\left(V_{\infty}-V_{i}\right)^{2}
$$

The thrust coefficient is defined to be the ratio of the thrust on the turbine disk to the freestream dynamic pressure multiplied by the area of the turbine disk. Applying the definition of the axial induction factor,

$$
a \equiv V_{i} / V_{\infty}
$$


the thrust coefficient can be written from Eq. (8) as

$$
C_{T} \equiv \frac{T}{\frac{1}{2} \rho V_{\infty}^{2} A_{t}}=4 \frac{V_{i}}{V_{\infty}}\left(1-\frac{V_{i}}{V_{\infty}}\right)=4 a(1-a)
$$

The power coefficient is defined as the ratio of the power extracted from the fluid to the power available in the undisturbed flowfield over the disk area. Therefore, using Eqs. (10) and (11) the power coefficient can be written as

$$
C_{P} \equiv \frac{P}{\frac{1}{2} \rho V_{\infty}^{3} A_{t}}=4 \frac{V_{i}}{V_{\infty}}\left(1-\frac{V_{i}}{V_{\infty}}\right)^{2}=4 a(1-a)^{2}
$$

Figure 2 shows a plot of the thrust and power coefficients as a function of the axial induction factor, $a$. If $a$ were an independent variable, it could be chosen to maximize the power coefficient of the actuator disk. This is the approach taken in the development of the Lanchester-Betz-Joukowski limit. The maximum of Eq. (13) occurs when $a=1 / 3$. Using this result in Eqs. (12) and (13) gives an upper limit on the power coefficient for an actuator disk as well as the corresponding thrust coefficient

$$
C_{P}=\frac{16}{27}, \quad C_{T}=\frac{8}{9}
$$

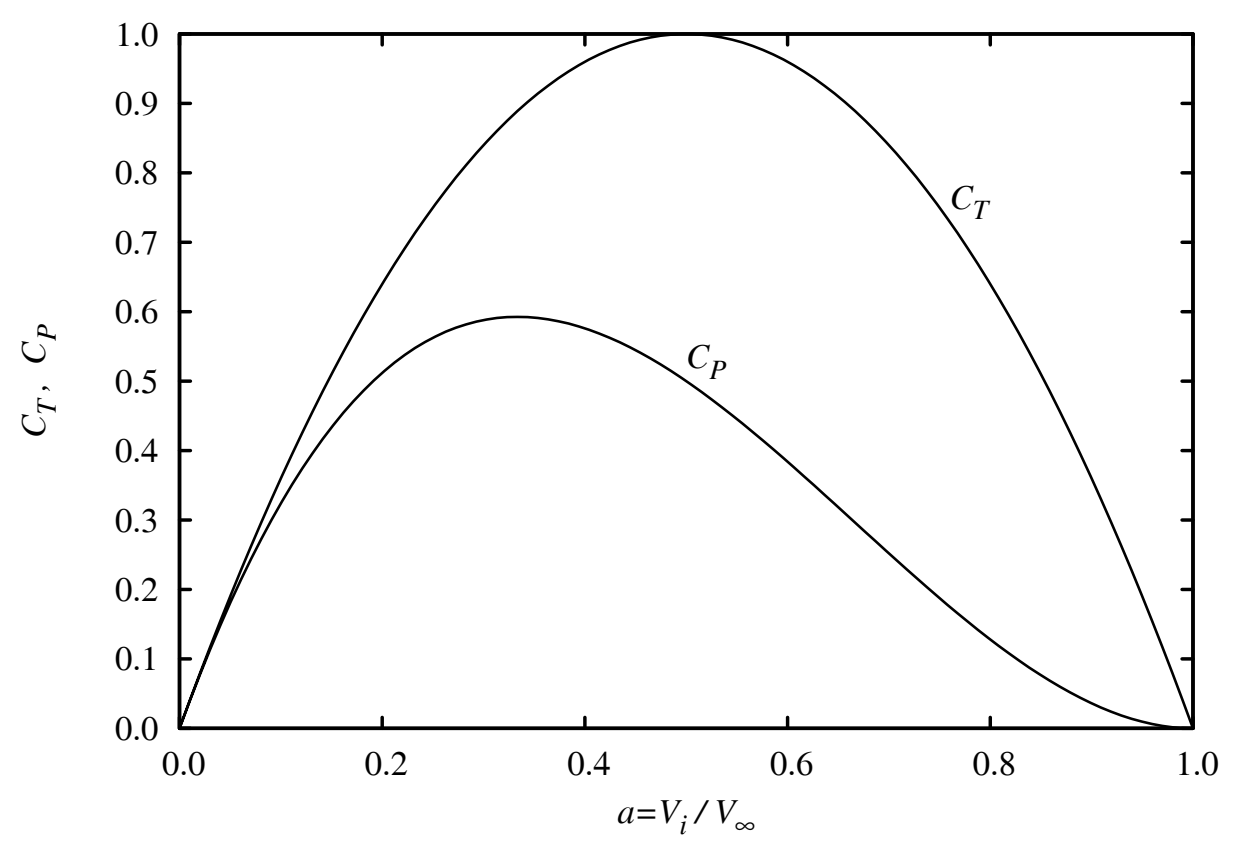

Figure 2. Thrust and power coefficients as a function of the axial induction factor.

An item of note that seems to be overlooked in the literature is that $a$ is not an independent variable. Clearly, $a$ is not a variable that the designer can choose just as the thrust or power coefficients are not variables that can be directly chosen by the designer. Rather $a, C_{T}$, and $C_{P}$ are all dependent on the design and operating conditions of the wind turbine and are related to each other through Eqs. (12) and (13). Equation (12) can be rearranged as a quadratic in $a$ to yield

$$
4 a^{2}-4 a+C_{T}=0
$$


Solving for $a$ in terms of the thrust coefficient yields two solutions.

$$
a=\frac{1}{2} \pm \frac{1}{2} \sqrt{1-C_{T}}
$$

To determine which of the two solutions is physically viable, it may help to study Fig. 2 in more detail. Recall that the axial induction factor, $a$, is defined as the ratio of the axial component of induced velocity at the turbine disk to the freestream velocity. Also recall from Fig. 1 that a positive value for the induced velocity is a velocity in the opposite direction as the freestream. The case of $a=0$ is equivalent to what could be termed a "perfectly" feathered turbine, which allows the fluid to pass through the turbine disk undisturbed. This would extract no power from the freestream, and in turn, produce no thrust as can be seen in Fig. 2. As the blades of the turbine are pitched and begin to extract power from the fluid, the axial induction factor increases and velocity is induced in the plane of the turbine disk opposite to the freestream. This increases both the thrust and power coefficients. Figure 2 shows that the maximum power coefficient is achieved when the axial induction factor reaches $a=1 / 3$. At that point, an increase in the induced velocity results in an increase in thrust and a decrease in power. If the induced velocity increases to onehalf the freestream velocity, the predicted thrust coefficient is equal to 1.0. Clearly, an increase in induced velocity beyond this point must result in an increase in thrust coefficient. However, this model predicts that for $a>1 / 2$, the thrust coefficient reduces with increasing axial induction factor. From Eq. (7), it can be seen that in the limit as the axial induction factor approaches one-half, the velocity in the ultimate slipstream approaches zero. In this limit, conservation of mass within the streamtube requires that the ultimate slipstream radius approach infinity. This is clearly not realistic. To further illustrate this point, note that the model predicts that the thrust and power coefficients are zero for an axial induction factor of unity. In this case, no fluid would pass through the turbine disk and the thrust would be clearly greater than zero. As a result of these model characteristics, this model can be applied only to the range $a<1 / 2$ and should be used with caution in the limit as $a$ approaches $1 / 2$.

It is also important to note that for $a<0$, the induced velocity is in the same direction as the freestream velocity and the power extracted from the flow becomes negative as can been seen from Eq. (13). For this operating condition, power is imparted to the flow through the actuator disk, which corresponds to the operating conditions of a propeller. As stated earlier, this model can be used to evaluate propeller performance as well as turbine performance. Because this paper focuses on the performance of wind turbines, results will be shown only for the range $0 \leq a \leq 1 / 2$.

Solving Eq. (15) for the only physically viable solution for $a$ gives

$$
a=\frac{1}{2}-\frac{1}{2} \sqrt{1-C_{T}}
$$

Using this in Eq. (13) gives a relationship between the power coefficient and the thrust coefficient

$$
C_{P}=C_{T}(1-a)=\frac{1}{2} C_{T}\left(1+\sqrt{1-C_{T}}\right)
$$

Although this classical model suffers from several unrealistic approximations, one major objection to these results has been the failure of the method to account for the rotation of the fluid within the slipstream. There appears to be no physical basis for neglecting slipstream rotation. Clearly, the power available in the freestream must be extracted by rotating the turbine. The torque will produce fluid rotation in the slipstream, and the power required to rotate the slipstream will affect the balance of energy. In the following analysis, we shall consider the effects of wake rotation.

\section{Momentum Theory Including Slipstream Rotation}

We shall now consider the incorporation of the angular momentum equation into the model hypothesized in the traditional momentum theory. Here we will continue with the assumptions of inviscid, incompressible flow, but we will allow for rotation of the fluid within the streamtube, which encloses the complete turbine disk as shown in Fig. 3. This streamtube is also assumed to extend infinitely far upstream from the turbine disk, to a plane where the static pressure is constant and equal to the freestream static pressure $p_{\infty}$. In this plane the axial component of 
velocity is the freestream velocity $V_{\infty}$, and there is no circumferential component of velocity. Likewise, the streamtube is assumed to extend infinitely far downstream from the turbine disk to a plane where the velocity in the slipstream is no longer changing in the axial direction. Consistent with the uniform flow assumption used in classical momentum theory, we will continue to assume uniform axial velocity but will now allow for a uniform angular velocity as well.

Because we wish to examine the effects of slipstream rotation on the results predicted by classical momentum theory, at first thought one might be inclined to continue with the assumption of uniform pressure, which is also imposed in classical momentum theory. However, this assumption is not consistent with rotation in the slipstream.

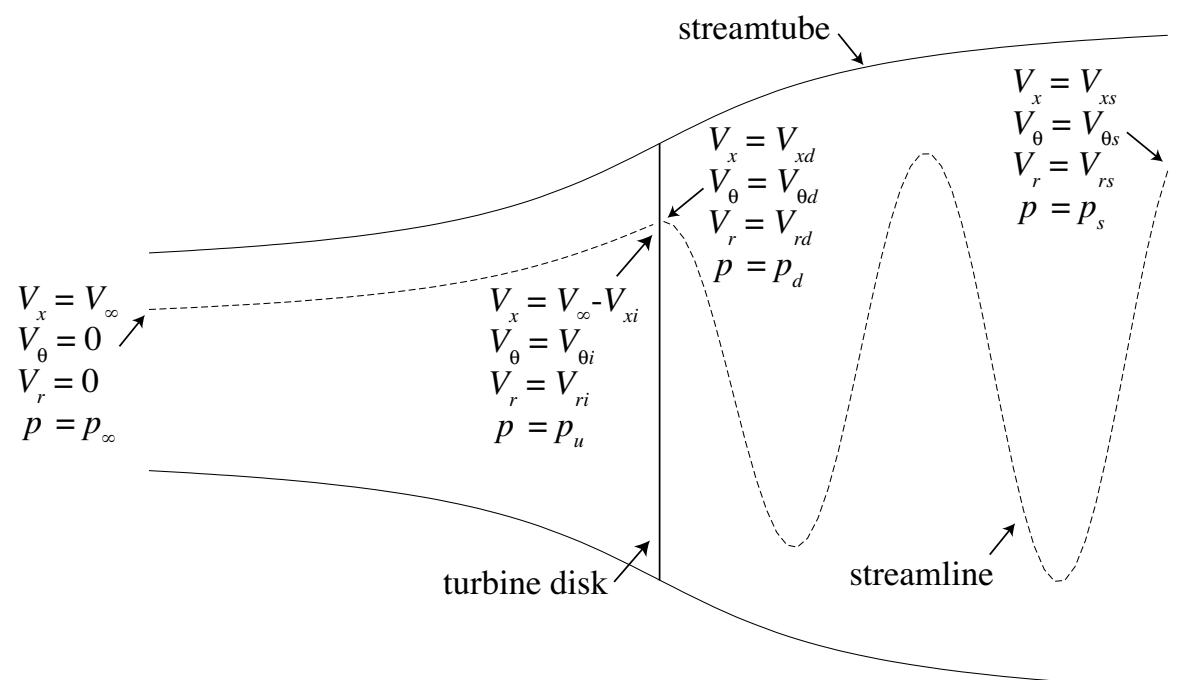

Figure 3. Momentum theory model for the pressures and velocities of the fluid that flows through the turbine disk including the effects of fluid rotation.

The solution obtained from classical propeller momentum theory does not satisfy the angular momentum equation. If we continue with the uniform pressure assumption from classical momentum theory while allowing for fluid rotation, we find that there is only one possible case for which the angular momentum equation together with the other laws of Newtonian mechanics can all be satisfied with a finite turbine rotational speed. That is the case of zero thrust. Because we are now allowing for fluid rotation in the streamtube and we wish to consider a turbine with finite thrust and power, we can no longer assume uniform pressure over each cross section of the streamtube. We must instead allow the pressure in the streamtube to be a function of the radial position $r$, as well as the axial position $x$.

On the near upstream side of the turbine disk, the axial component of fluid velocity is equal to the freestream velocity minus the axial component of induced velocity $V_{\infty}-V_{x i}$. On the near downstream side of the turbine disk, the axial component of fluid velocity is designated $V_{x d}$. The circumferential component of velocity on the near upstream side of the turbine disk is simply the circumferential component of the induced velocity $V_{\theta i}$, and the circumferential component of velocity on the near downstream side of the turbine disk is denoted as $V_{\theta d}$. Likewise, the radial velocity component on the near upstream side of the turbine disk is simply the radial component of the induced velocity $V_{r i}$, and the radial velocity component on the near downstream side of the turbine disk is denoted as $V_{r d}$.

The circumferential component of fluid velocity just upstream from the turbine disk can be deduced by applying the angular momentum equation to the section of streamtube that is upstream from the turbine disk. Because there is no torque acting on this section of the streamtube, there can be no change in the angular momentum of the fluid as it passes through this section. Because the fluid has no angular momentum far upstream from the turbine disk, it cannot have angular momentum when it reaches the upstream side of the turbine disk. This requires

$$
V_{\theta i}=0
$$


As a result of torque applied to the turbine, angular momentum is imparted to the fluid as it flows through the turbine disk. In the section of the streamtube downstream of the turbine disk, the angular momentum flux must again remain constant since there is no torque applied to this section of the streamtube. At first thought, one might be led to conclude that the angular velocity remains constant in the downstream section of the streamtube, just as it did in the upstream section. However, this is not the case. The angular velocity remains constant in the upstream section of the streamtube only because the angular velocity is zero in the freestream. As fluid flows from the region far upstream toward the high-pressure side of the turbine disk, the axial component of velocity is decreased due to the pressure increase. Likewise, as the fluid flows away from the low-pressure side of the turbine disk, the axial component of velocity continues to decrease as a result of the pressure difference between the downstream side of the turbine disk and the far slipstream. This deceleration causes the streamtube enclosing the slipstream to expand in diameter with increasing distance downstream from the turbine disk as shown in Fig. 3. Since the diameter of the streamtube increases and the angular momentum flux for the fluid remains constant, the angular velocity of the fluid in the slipstream must decrease along with the axial component of velocity as it moves away from the downstream side of the turbine disk. To evaluate the induced velocity, we consider the flow along the streamline shown in Fig. 3.

The axial and radial components of fluid velocity just downstream from the turbine disk is related to the axial and radial components on the upstream side through mass conservation. If we use the notation shown in Fig. 3 and apply conservation of mass across any radial segment of the turbine disk, for the assumed incompressible flow we have

$$
\begin{aligned}
& V_{x d}=V_{\infty}-V_{x i} \\
& V_{r d}=V_{r i}
\end{aligned}
$$

The pressure and velocity just upstream from the turbine disk are related to the freestream pressure and velocity through Bernoulli's equation. Applying Bernoulli's equation along the streamline shown in Fig. 3 and using Eqs. (19) and (20), upstream from the turbine disk we obtain

$$
\frac{p_{\infty}}{\rho}+\frac{V_{\infty}^{2}}{2}=\frac{p_{u}}{\rho}+\frac{V_{x d}^{2}+V_{r d}^{2}}{2}
$$

Here we see that because there is no rotation of the fluid in the upstream section of the streamtube the uniform flow approximation requires uniform pressure upstream from the turbine disk. Applying Bernoulli's equation to a streamline on the aft side of the turbine disk beginning at a point just behind the turbine and extending to a point in the far slipstream gives

$$
\frac{p_{s}}{\rho}+\frac{V_{x s}^{2}+V_{\theta s}^{2}+V_{r s}^{2}}{2}=\frac{p_{d}}{\rho}+\frac{V_{x d}^{2}+V_{\theta d}^{2}+V_{r d}^{2}}{2}
$$

where $V_{x s}, V_{\theta s}$, and $V_{r s}$ are the axial, circumferential, and radial components of velocity in the ultimate slipstream.

Subtracting Eq. (21) from Eq. (22) gives

$$
p_{s}-p_{\infty}+\frac{1}{2} \rho\left(V_{x s}^{2}+V_{\theta s}^{2}+V_{r s}^{2}-V_{\infty}^{2}\right)=p_{d}-p_{u}+\frac{1}{2} \rho V_{\theta d}^{2}
$$

From the uniform flow approximation, the circumferential component of velocity in the ultimate slipstream can be written as the product of the angular velocity and the local radius.

$$
V_{\theta s}=\omega_{s} r_{s}
$$

Using this in Eq. (23) gives

$$
p_{s}-p_{\infty}+\frac{1}{2} \rho\left(V_{x s}^{2}+\omega_{s}^{2} r_{s}^{2}+V_{r s}^{2}-V_{\infty}^{2}\right)=p_{d}-p_{u}+\frac{1}{2} \rho V_{\theta d}^{2}
$$


where $\omega_{s}$ and $r_{s}$ are the angular velocity and radial coordinate at the location of the streamline far downstream from the turbine disk. At this point, other developments attempting to account for rotational effects have commonly employed Bernoulli's equation across the rotating disk. ${ }^{15}$ This is justified on the grounds that the work done by the turbine disk due to lift is normal to the local velocity along the blades. ${ }^{16}$ However, a different approach is taken here.

In the ultimate slipstream, the flow properties are independent of axial position and circumferential position, and the continuity equation requires that

$$
\frac{\partial\left(r_{s} V_{r s}\right)}{\partial r_{s}}=0
$$

The three components of the momentum equation in the slipstream reduce to

$$
\begin{gathered}
V_{r s} \frac{\partial V_{r s}}{\partial r_{s}}-\frac{V_{\theta s}^{2}}{r_{s}}=-\frac{1}{\rho} \frac{d p_{s}}{d r_{s}} \\
V_{r s} \frac{\partial V_{\theta s}}{\partial r_{s}}+\frac{V_{r s} V_{\theta s}}{r_{s}}=0 \\
V_{r s} \frac{\partial V_{x s}}{\partial r_{s}}=0
\end{gathered}
$$

Solving Eq. (26) subject to the outer boundary condition which specifies that there is no radial component of velocity at the edge of the ultimate slipstream gives

$$
V_{r s}=0
$$

As a result of Eq. (30), Eqs. (28) and (29) are identically satisfied. Applying Eq. (30) to Eq. (27) gives

$$
\frac{d p_{s}}{d r_{s}}=\rho \frac{V_{\theta s}^{2}}{r_{s}}=\rho \omega_{s}^{2} r_{s}
$$

Equation (31) shows that the radial pressure gradient in the ultimate slipstream is equal to the angular momentum in the ultimate slipstream. Therefore, if the angular momentum of the slipstream is nonzero, a nonzero pressure gradient must exist in order to balance the radial forces as asserted by Sharpe. ${ }^{16}$ At the outer edge of the slipstream, $r_{s}=R_{s}$, the pressure must be equal to the freestream pressure. Integrating Eq. (31) and applying this boundary condition gives

$$
\begin{aligned}
& \int_{p_{s}}^{p_{\infty}} d p_{s}=\int_{r_{s}}^{R_{s}} \rho \omega_{s}^{2} r_{s} d r_{s} \\
& p_{s}=p_{\infty}-\frac{\rho \omega_{s}^{2}}{2}\left(R_{s}^{2}-r_{s}^{2}\right)
\end{aligned}
$$

Using Eqs. (30) and (32) in Eq. (25) gives

$$
\rho \omega_{s}^{2}\left(r_{s}^{2}-\frac{R_{s}^{2}}{2}\right)+\frac{1}{2} \rho\left(V_{x s}^{2}-V_{\infty}^{2}\right)=p_{d}-p_{u}+\frac{1}{2} \rho V_{\theta d}^{2}
$$

Applying mass conservation to the flow inside the stream surface that contains the streamline under consideration from the upwind surface of the turbine to the ultimate slipstream gives 


$$
\pi r_{t}^{2} \rho\left(V_{\infty}-V_{x i}\right)=\pi r_{s}^{2} \rho V_{x s}
$$

where $r_{t}$ is the radial position of the streamline at the turbine. Rearranging gives

$$
r_{s}^{2}=r_{t}^{2} \frac{\left(V_{\infty}-V_{x i}\right)}{V_{x s}}
$$

Using this in Eq. (33) gives

$$
\rho \omega_{s}^{2}\left(r_{t}^{2}-\frac{R_{t}^{2}}{2}\right) \frac{\left(V_{\infty}-V_{x i}\right)}{V_{x s}}+\frac{1}{2} \rho\left(V_{x s}^{2}-V_{\infty}^{2}\right)=p_{d}-p_{u}+\frac{1}{2} \rho V_{\theta d}^{2}
$$

where $R_{t}$ is the outer radius of the turbine. Because angular momentum stays constant downstream from the turbine,

$$
r_{t} V_{\theta d}=r_{s} V_{\theta s}=r_{s}^{2} \omega_{s}
$$

Combining Eqs. (35) and Eq. (37) gives

$$
V_{\theta d}=\frac{\left(V_{\infty}-V_{x i}\right)}{V_{x s}} r_{t} \omega_{s}
$$

Using this relationship in Eq. (36) and rearranging yields

$$
p_{d}-p_{u}=\rho \omega_{s}^{2}\left[\left(1-\frac{V_{\infty}-V_{x i}}{2 V_{x s}}\right) r_{t}^{2}-\frac{R_{t}^{2}}{2}\right] \frac{\left(V_{\infty}-V_{x i}\right)}{V_{x s}}+\frac{1}{2} \rho\left(V_{x s}^{2}-V_{\infty}^{2}\right)
$$

The axial momentum deficit, or the thrust, can be written in terms of the axial momentum equation relating the properties in the ultimate slipstream to those in the freestream

$$
T=\int_{A_{s}}\left(p_{\infty}-p_{s}\right) d A_{s}+\int_{A_{s}}\left(V_{\infty}-V_{x s}\right) d \dot{m}
$$

Using Eq. (32) gives

$$
T=\int_{r_{s}=0}^{R_{s}} \frac{\rho \omega_{s}^{2}}{2}\left(R_{s}^{2}-r_{s}^{2}\right) 2 \pi r_{s} d r_{s}+\int_{r_{s}=0}^{R_{s}}\left(V_{\infty}-V_{x s}\right) \rho V_{x s} 2 \pi r_{s} d r_{s}
$$

Because we have assumed that the angular rate and the axial component of velocity of the slipstream are constant across the slipstream, this can be integrated to give

$$
T=\frac{\pi \rho \omega_{s}^{2} R_{s}^{4}}{4}+\pi \rho R_{s}^{2} V_{x s}\left(V_{\infty}-V_{x s}\right)
$$

Using Eq. (35), this can also be written as

$$
T=\pi \rho R_{t}^{2}\left[\frac{\omega_{s}^{2} R_{t}^{2}}{4}\left(\frac{V_{\infty}-V_{x i}}{V_{x s}}\right)^{2}+\left(V_{\infty}-V_{x i}\right)\left(V_{\infty}-V_{x s}\right)\right]
$$


The thrust can be written alternately in terms of the pressure difference across the plane of the turbine disk.

$$
T=\int_{A_{t}}\left(p_{u}-p_{d}\right) d A_{t}=\int_{r_{t}=0}^{R_{t}}\left(p_{u}-p_{d}\right) 2 \pi r_{t} d r_{t}
$$

Using Eq. (39) in Eq. (44) and integrating yields

$$
T=\pi \rho R_{t}^{2}\left[\omega_{s}^{2} R_{t}^{2}\left(\frac{V_{\infty}-V_{x i}}{2 V_{x s}}\right)^{2}-\frac{V_{x s}^{2}}{2}+\frac{V_{\infty}^{2}}{2}\right]
$$

Equating the expressions for thrust given in Eqs. (43) and (45) gives the relation

$$
V_{x s}=V_{\infty}-2 V_{x i}
$$

Note that this is the same relation developed in Eq. (7) which was developed assuming no rotation in the wake.

As the fluid exerts a torque on the turbine, an equal and opposite torque is imparted to the fluid by the turbine. This reaction torque causes the flow in the wake to rotate in the opposite direction as the turbine. The torque on the turbine is related to the rotation in the ultimate slipstream through the angular momentum equation

$$
Q=-\int_{A_{s}} r_{s} V_{\theta s} d \dot{m}=-\int_{r_{s}=0}^{R_{s}} r_{s}^{2} \omega_{s}\left(\rho V_{x s} 2 \pi r_{s} d r_{s}\right)=-\frac{\pi}{2} \rho V_{x s} \omega_{s} R_{s}^{4}
$$

Applying Eq. (35) gives

$$
Q=-\pi \rho \omega_{s} R_{t}^{4}\left(V_{\infty}-V_{x i}\right)^{2} /\left(2 V_{x s}\right)
$$

The power extracted from the fluid by the turbine must satisfy the first law of thermodynamics

$$
P=\int_{A_{s}}\left(h_{\infty}-h_{s}+\frac{V_{\infty}^{2}}{2}-\frac{V_{x s}^{2}+V_{\theta s}^{2}}{2}\right) d \dot{m}
$$

For the assumed incompressible flow, this reduces to

$$
P=\int_{r_{s}=0}^{R_{s}}\left(\frac{p_{\infty}}{\rho}-\frac{p_{s}}{\rho}+\frac{V_{\infty}^{2}}{2}-\frac{V_{x s}^{2}+\omega_{s}^{2} r_{s}^{2}}{2}\right) \rho V_{x s} 2 \pi r_{s} d r_{s}
$$

Applying Eq. (32), integrating, and applying Eq. (35) to the result gives

$$
P=\pi \rho R_{t}^{2}\left(V_{\infty}-V_{x i}\right)\left(\frac{V_{\infty}^{2}}{2}-\frac{V_{x s}^{2}}{2}\right)
$$

The turbine extracts power from the fluid through the torque applied to the turbine given in Eq. (48). Multiplying Eq. (48) by the angular velocity of the turbine disk gives the brake power extracted from the fluid by the turbine. The brake power must be equal to the power given in Eq. (51). Multiplying the right-hand side of Eq. (48) by the angular velocity of the turbine disk, setting it equal to the right-hand side of Eq. (51), and applying the relation in Eq. (46) gives 


$$
\omega_{s}=-\frac{4\left(V_{\infty}-2 V_{x i}\right) V_{x i}}{\omega_{t} R_{t}^{2}}
$$

Applying Eqs. (52) and (46) to either Eq. (43) or (45) gives an expression for the thrust

$$
T=2 \pi \rho\left(V_{\infty}-V_{x i}\right) V_{x i}\left[\frac{2\left(V_{\infty}-V_{x i}\right) V_{x i}}{\omega_{t}^{2}}+R_{t}^{2}\right]
$$

Likewise, applying Eq. (46) to Eq. (51) gives the power extracted from the fluid by the turbine

$$
P=2 \pi \rho R_{t}^{2}\left(V_{\infty}-V_{x i}\right)^{2} V_{x i}
$$

Defining $\lambda \equiv R_{t} \omega_{t} / V_{\infty}$ as the turbine tip-speed ratio and applying the definition for the axial induction factor $a \equiv V_{x i} / V_{\infty}$, the thrust coefficient can be written as

$$
C_{T} \equiv \frac{T}{\frac{1}{2} \rho V_{\infty}^{2} A_{t}}=4 \frac{V_{x i}}{V_{\infty}}\left(\frac{V_{\infty}}{V_{\infty}}-\frac{V_{x i}}{V_{\infty}}\right)\left[\frac{2 V_{\infty}^{2} \frac{V_{x i}}{V_{\infty}}\left(\frac{V_{\infty}}{V_{\infty}}-\frac{V_{x i}}{V_{\infty}}\right)}{R_{t}^{2} \omega_{t}^{2}}+1\right]=4 a(1-a)\left[\frac{2 a(1-a)}{\lambda^{2}}+1\right]
$$

Note that this relationship between the thrust coefficient and the axial induction factor approaches that given in Eq. (12) as the tip-speed ratio approaches infinity. The power coefficient can be written as

$$
C_{P} \equiv \frac{P}{\frac{1}{2} \rho V_{\infty}^{3} A_{t}}=4 \frac{V_{x i}}{V_{\infty}}\left(1-\frac{V_{x i}}{V_{\infty}}\right)^{2}=4 a(1-a)^{2}
$$

Figure 4 shows the power coefficient as a function of $a$ as well as the thrust coefficient for several tip-speed ratios as a function of $a$. Just as in the classical momentum theory model, physics imposes conditions such that this model is constrained to solutions where $a \leq 1 / 2$. For the case of a wind turbine, the axial induction factor must be positive. Therefore, the following results are reported only for $0 \leq a \leq 1 / 2$.

Note that the relationship between the power coefficient and the axial induction factor is identical to that obtained from the classical momentum theory ignoring rotational effects and given in Eq. (13). Again, Eq. (56) predicts a maximum of $C_{P}=16 / 27$ at an axial induction factor of $a=1 / 3$. The Lanchester-Betz-Joukowski limit was developed by treating $a$ as an independent variable and taking the maximum of the power coefficient given in Eq. (13). Because Eq. (56) is identical to Eq. (13), using the same method to evaluate the ideal efficiency of a wind turbine including the effects of rotation in the slipstream gives the same result as when the rotation is ignored. Other methods for defining the efficiency of a wind turbine have been suggested. ${ }^{18,21}$ However, the efficiency used in the development of the Lanchester-Betz-Joukowski limit is the power coefficient defined in Eq. (56). Using this as the definition for the efficiency of a wind turbine and treating $a$ as an independent variable, we see that accounting for the rotation of the flow in the slipstream does not change this upper limit of $C_{P}=16 / 27$. This supports the claims of others ${ }^{25}$ who used different assumptions to reach the same conclusion.

Conversely, the method presented here yields a solution for the thrust coefficient that is a function of tip-speed ratio while the classical momentum theory predicts that the thrust is independent of tip-speed ratio. Thus we see that the change in the energy balance caused by the rotational momentum in the slipstream is manifest through a change in thrust coefficient rather than a change in power coefficient for a given axial induction factor. Although the magnitude of the thrust coefficient is less critical in wind turbine design than the power coefficient, the estimate for the thrust coefficient resulting from neglecting the slipstream effects is not conservative. Therefore, designing the tower of a wind turbine to withstand the thrust force predicted by the classical momentum theory could result in structural failure. 


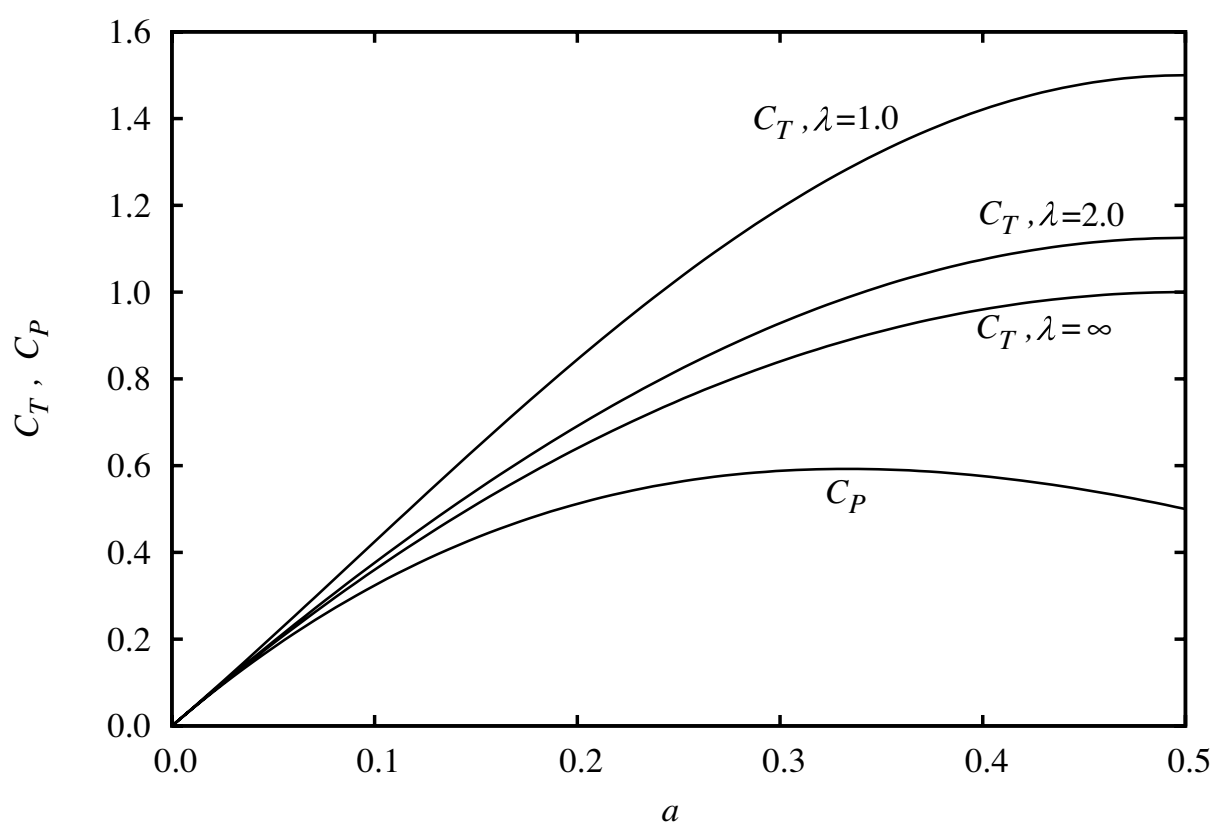

Figure 4. Power coefficient and thrust coefficient as a function of axial induction factor and tip-speed ratio.

\section{Turbine Performance}

Better insight into the effects of slipstream rotation can be obtained by considering the results of this model in terms of the independent variables of the wind turbine design and operation. The power extracted from the freestream by the turbine is transferred to a generator through a shaft. The generator converts the power transferred from the shaft into electrical energy that can be connected to the power grid or stored for future use. The efficiency of the generator in converting brake power to electrical power is dependent on the rotational speed of the shaft and the torque applied to the shaft. Therefore, two important independent variables of the design are the tip-speed ratio and the torque coefficient of the turbine. Many modern turbines are designed so that the tip-speed ratio and torque can be controlled by using variable pitch blades, yawing the turbine, or applying drag devices. Thus, the results of a wind turbine model may be more useful when viewed in terms of the tip-speed ratio and the torque coefficient.

Using Eqs. (46) and (52) in Eq. (48) gives an expression for the torque

$$
Q=2 \pi \rho R_{t}^{2} \frac{\left(V_{\infty}-V_{i}\right)^{2} V_{i}}{\omega_{t}}
$$

The torque coefficient for a wind turbine is commonly defined as

$$
C_{Q} \equiv \frac{Q}{\frac{1}{2} \rho \pi V_{\infty}^{2} R_{t}^{3}}
$$

Using this definition, Eq. (57) can be rearranged and written in nondimensional form as

$$
C_{Q}=\frac{4 a(1-a)^{2}}{\lambda}
$$

Although the result shown in Eq. (59) is written as if the torque coefficient were the dependent variable, the importance of this result is that it displays the relationship between the dependent variable, $a$, and the two independent variables, $C_{Q}$ and $\lambda$. This relationship can now be applied to show the relationships between other dependent variables and the independent variables. 


\section{A. Power Coefficient}

Combining Eqs. (56) and (59) to eliminate the axial induction factor gives a relationship for the power coefficient in terms of the torque coefficient and the tip-speed ratio

$$
C_{P}=C_{Q} \lambda
$$

Although this relationship has been rigorously derived in this development, it is a rather straight forward relationship between tip-speed ratio, torque coefficient, and power coefficient and is commonly used even in developments for which the rotation in the slipstream is neglected. Because the theoretical maximum power coefficient is $C_{P}=16 / 27$, using this in Eq. (60) gives an ideal relationship between the torque coefficient and tip-speed ratio

$$
\lambda=16 /\left(27 C_{Q}\right)
$$

According to this model, if this relation between the tip-speed ratio and torque coefficient is maintained, the power coefficient will be at a maximum of $C_{P}=16 / 27$. Figure 5 shows the power coefficient as a function of tip-speed ratio and torque coefficient. The ideal operating condition or upper limit for the power coefficient is also included in the figure.

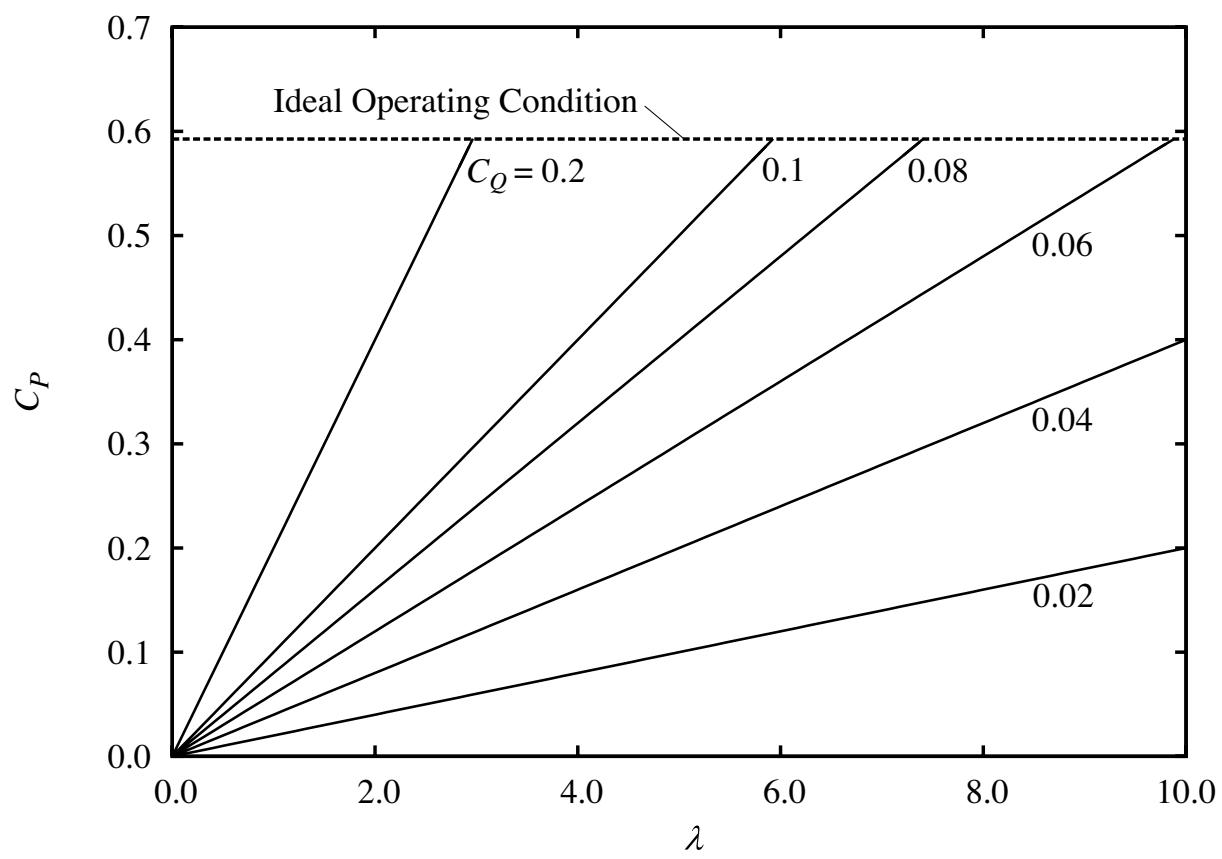

Figure 5. Power coefficient as a function of tip-speed ratio and torque coefficient.

\section{B. Axial Induction Factor}

Recall that the Lanchester-Betz-Joukowski limit was obtained by treating the axial induction factor $a$ as an independent variable and solving for the value of $a$ that provided the maximum power coefficient. However, as stated previously, the axial induction factor is not an independent variable. Rather it is a property of the flow that is dependent on the operating conditions of the turbine. Therefore, it is helpful to find an expression for the axial induction factor in terms of the torque coefficient and tip-speed ratio, which are two independent variables. We see from Eqs. (56) and (59) that both the power and torque coefficients are cubic functions of the axial induction factor. To simplify the following analysis, we will find an expression for the axial induction factor in terms of the power coefficient, and then apply the relationship $C_{P}=C_{Q} \lambda$. Equation (56) can be rearranged to yield 


$$
a^{3}-2 a^{2}+a-C_{P} / 4=0
$$

The roots of Eq. (62) yield the axial induction factor as a function of the power coefficient. In order to obtain the roots of this equation, it is convenient to use the change of variables

$$
u \equiv a-2 / 3
$$

Using Eq. (63) in Eq. (62) and rearranging yields

$$
u^{3}-\frac{1}{3} u+\frac{2}{27}-\frac{C_{P}}{4}=0
$$

In general, the three roots of Eq. (64) are obtained from

$$
u=A+B, \quad-\frac{A+B}{2}+\frac{A-B}{2} \sqrt{-3}, \quad-\frac{A+B}{2}-\frac{A-B}{2} \sqrt{-3}
$$

where

$$
A \equiv \frac{1}{2} \sqrt[3]{C_{p}-\frac{8}{27}+\sqrt{\left(C_{p}-\frac{8}{27}\right)^{2}-\left(\frac{8}{27}\right)^{2}}}, \quad B \equiv \frac{1}{2} \sqrt[3]{C_{p}-\frac{8}{27}-\sqrt{\left(C_{p}-\frac{8}{27}\right)^{2}-\left(\frac{8}{27}\right)^{2}}}
$$

Notice from Eqs. (65) and (66) that for the case $C_{P}=16 / 27$, there are three real roots; one distinct root at $u=2 / 3$, and two identical roots at $u=-1 / 3$. From the definition in Eq. (63), the axial induction factor for the distinct root is $a=4 / 3$ and that for the two identical roots is $a=1 / 3$. Because the distinct root corresponds to an axial induction factor greater than $a=1 / 2$, the only physically viable solution to Eq. (62) for the case $C_{P}=16 / 27$ is $a=1 / 3$.

For the case $C_{P}>16 / 27$, Eq. (65) yields one real root and two conjugate complex roots. The real root always results in $u>2 / 3$, which from Eq. (63) always corresponds to an axial induction factor $a>4 / 3$. Hence there are no physically viable solutions to Eq. (62) for the case $C_{P}>16 / 27$.

For the case $0 \leq C_{P} \leq 16 / 27$, Eq. (65) yields three distinct real roots. In this case, a trigonometric solution may be more useful

$$
\begin{aligned}
u=\frac{2}{3} \cos \left(\frac{\phi}{3}\right), & \frac{2}{3} \cos \left(\frac{\phi}{3}+\frac{2 \pi}{3}\right), \frac{2}{3} \cos \left(\frac{\phi}{3}+\frac{4 \pi}{3}\right) \\
\phi & \equiv \cos ^{-1}\left(\frac{27 C_{p}}{8}-1\right)
\end{aligned}
$$

From the definition in Eq. (63), the first of the three roots given in Eq. (67) gives

$$
\pi \geq \phi \geq 0, \quad \frac{1}{3} \leq u \leq \frac{2}{3}, \quad 1 \leq a \leq \frac{4}{3}, \quad \text { for } \quad 0 \leq C_{p} \leq \frac{16}{27}
$$

Similarly, the second of the three roots given in Eq. (67) yields

$$
\pi \geq \phi \geq 0, \quad-\frac{2}{3} \leq u \leq-\frac{1}{3}, \quad 0 \leq a \leq \frac{1}{3}, \quad \text { for } \quad 0 \leq C_{p} \leq \frac{16}{27}
$$

and the third of the three roots results in 


$$
\begin{aligned}
& \pi \geq \phi \geq \cos ^{-1}\left(\frac{11}{16}\right), \quad \frac{1}{3} \geq u \geq-\frac{1}{6}, \quad 1 \geq a \geq \frac{1}{2}, \quad \text { for } \quad 0 \leq C_{p} \leq \frac{1}{2} \\
& \cos ^{-1}\left(\frac{11}{16}\right) \geq \phi \geq 0, \quad-\frac{1}{6} \geq u \geq-\frac{1}{3}, \quad \frac{1}{2} \geq a \geq \frac{1}{3}, \quad \text { for } \quad \frac{1}{2} \leq C_{p} \leq \frac{16}{27}
\end{aligned}
$$

From Eqs. (68) - (70), we see that the first root in Eq. (67) provides no physically viable solutions to Eq. (62); the second of the three roots is physically valid over the entire range $0 \leq C_{P} \leq 16 / 27$; and the third root is physically valid only over the range $1 / 2 \leq C_{P} \leq 16 / 27$. Hence, applying the relation $C_{P}=C_{Q} \lambda$, the only physically viable solutions to Eq. (62) can be expressed as

$$
a= \begin{cases}\frac{2}{3} \cos \left(\frac{\phi}{3}+\frac{2 \pi}{3}\right)+\frac{2}{3}, & \text { for } 0 \leq C_{Q} \lambda \leq \frac{1}{2} \\ \frac{2}{3} \cos \left(\frac{\phi}{3}+\frac{2 \pi}{3}\right)+\frac{2}{3}, & \frac{2}{3} \cos \left(\frac{\phi}{3}+\frac{4 \pi}{3}\right)+\frac{2}{3}, \quad \text { for } \frac{1}{2} \leq C_{Q} \lambda \leq \frac{16}{27}\end{cases}
$$

where

$$
\phi \equiv \cos ^{-1}\left(27 C_{Q} \lambda / 8-1\right)
$$

Equations (71) and (72) allow the axial induction factor to be evaluated from known values of the tip-speed ratio and torque coefficient. Figure 6 shows the axial induction factor as a function of tip-speed ratio for several values of torque coefficient. Note from Eq. (71) and Fig. 6 that there are two solutions for $1 / 2 \leq C_{Q} \lambda<16 / 27$. The ideal operating conditions occur when $a=1 / 3$ and are indicated in the figure.

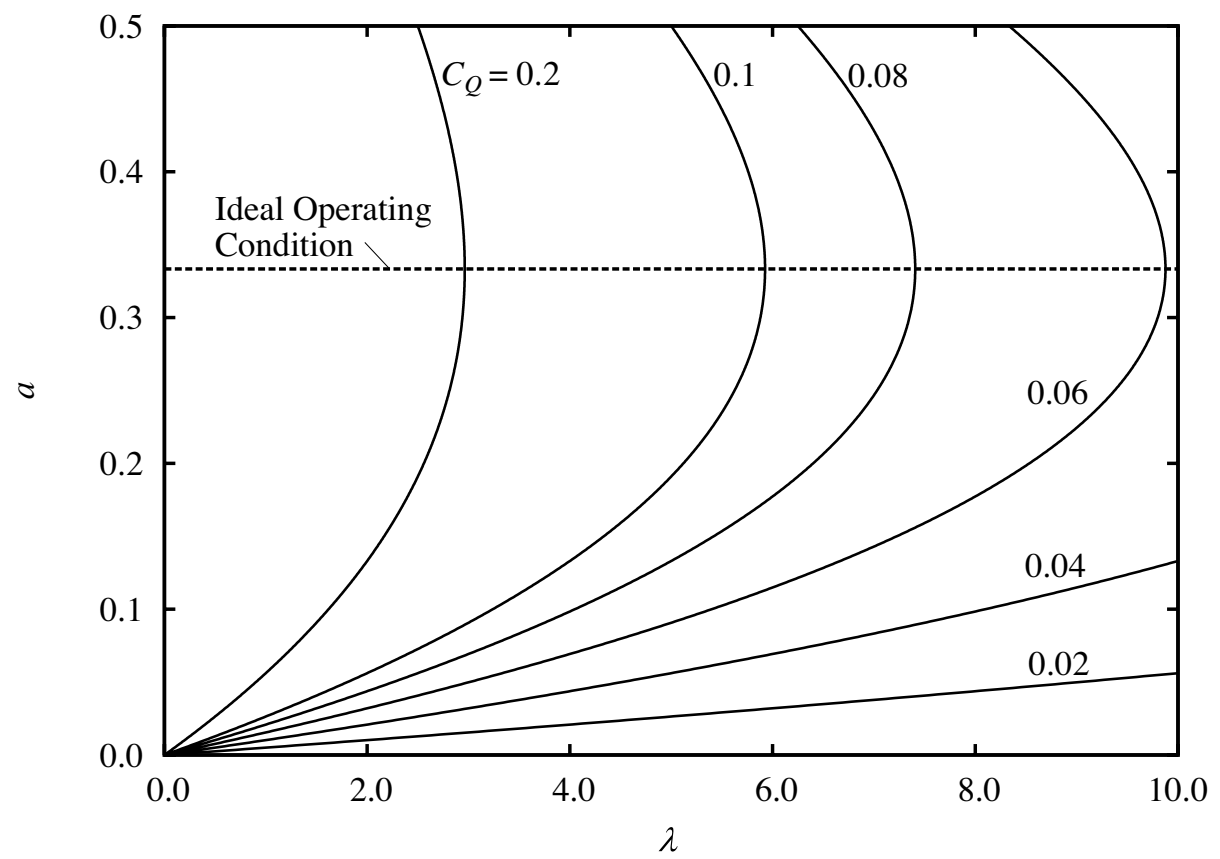

Figure 6. Axial induction factor as a function of tip-speed ratio and torque coefficient.

As stated earlier, Eq. (56) predicts that the theoretical maximum for the power coefficient is reached when $a=1 / 3$. Using this value for the axial induction factor on the left-hand side of Eq. (71) gives the ideal relationship between the tip-speed ratio and the torque coefficient given in Eq. (61). 


\section{Thrust Coefficient}

Once the axial induction factor for a given tip-speed ratio and torque coefficient is found, the result can be used in Eq. (55) to evaluate the thrust coefficient as a function of tip-speed ratio and torque coefficient. Comparing Eqs. (12) and (55) it can be seen that the prediction for thrust including the effects of slipstream rotation is always greater than the thrust predicted by neglecting slipstream rotation for finite tip-speed ratios. Because all wind turbines operate at finite tip-speed ratios, ignoring the effects of slipstream rotation is not a conservative approach for thrust estimation, and Eq. (55) should always be used instead of Eq. (12). The difference between the two estimates for thrust is particularly important at low tip-speed ratios.

Figure 7 shows results for the thrust coefficient predicted by this model. Again, note the two solutions for $1 / 2 \leq C_{Q} \lambda<16 / 27$. The thrust coefficient at ideal operating conditions can be found by using the optimum value $a=1 / 3$ in Eq. (55) which yields

$$
C_{T}=\frac{8}{9}\left(\frac{4}{9 \lambda^{2}}+1\right)
$$

This relation is also shown in Fig. 7. Note that high tip-speed ratios and low torque coefficients yield lower thrust coefficients. As the tip-speed ratio becomes large, the optimal thrust coefficient approaches that predicted by the classical momentum theory which neglects slipstream rotation, $C_{T}=8 / 9$. At low tip-speed ratios and high torque coefficients, the ideal-operating-condition thrust coefficient deviates significantly from that obtained from ignoring the slipstream-rotation effects.

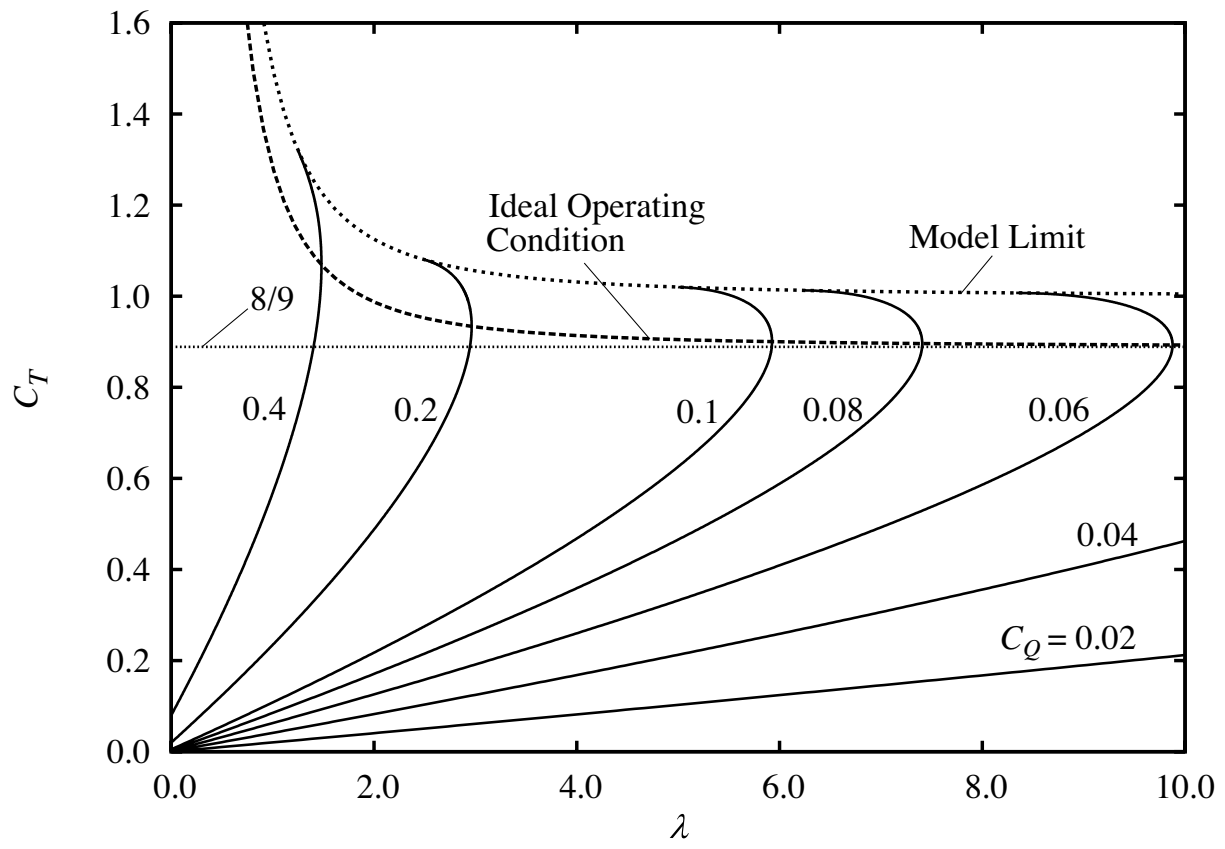

Figure 7. Thrust coefficient as a function of tip-speed ratio and torque coefficient.

\section{Circumferential Induction Factor}

One final note relating this work to previous methods for estimating the influence of the rotation in the wake may be in order. In previous developments it has been customary to define a circumferential induction factor at the turbine disk and to show how it is related to the axial induction factor. The circumferential induction factor is commonly defined as

$$
a^{\prime} \equiv \frac{V_{\theta p}}{\omega_{t} r_{t}}
$$


where $V_{\theta p}$ is the circumferential component of induced velocity directly in the plane of the turbine disk. This circumferential component of velocity has traditionally been accepted to be equal to half of the circumferential component of velocity at the downstream side of the turbine disk

$$
V_{\theta p}=\frac{1}{2} V_{\theta d}
$$

This is justified on the grounds that the turbine disk represents a sheet of vorticity inducing a step change in the circumferential component of velocity, which reaches half its total magnitude at the plane of the turbine disk. Combining Eqs. (74) and (75) and using this in Eq. (38) gives

$$
V_{\theta d}=2 a^{\prime} \omega_{t} r_{t}=\frac{\left(V_{\infty}-V_{x i}\right)}{V_{x s}} r_{t} \omega_{s}
$$

Using Eqs. (46) and (52) and applying the definitions for the axial induction factor and tip-speed ratio gives an expression for the circumferential induction factor

$$
a^{\prime}=-\frac{2 a(1-a)}{\lambda^{2}}
$$

Equations (71) and (72) can be used to evaluate the axial induction factor as a function of the tip-speed ratio and torque coefficient. These results can be used in Eq. (77) to evaluate the circumferential induction factor as a function of the tip-speed ratio and torque coefficient. The circumferential induction factor at the ideal operating condition is found by using the optimum value $a=1 / 3$ in Eq. (77). This yields

$$
a^{\prime}=-\frac{4}{9 \lambda^{2}}
$$

Figure 8 shows the relationship given in Eq. (77) as a function of tip-speed ratio and torque coefficient as well as the ideal operating condition given in Eq. (78). Note that the axes are logarithmically scaled.

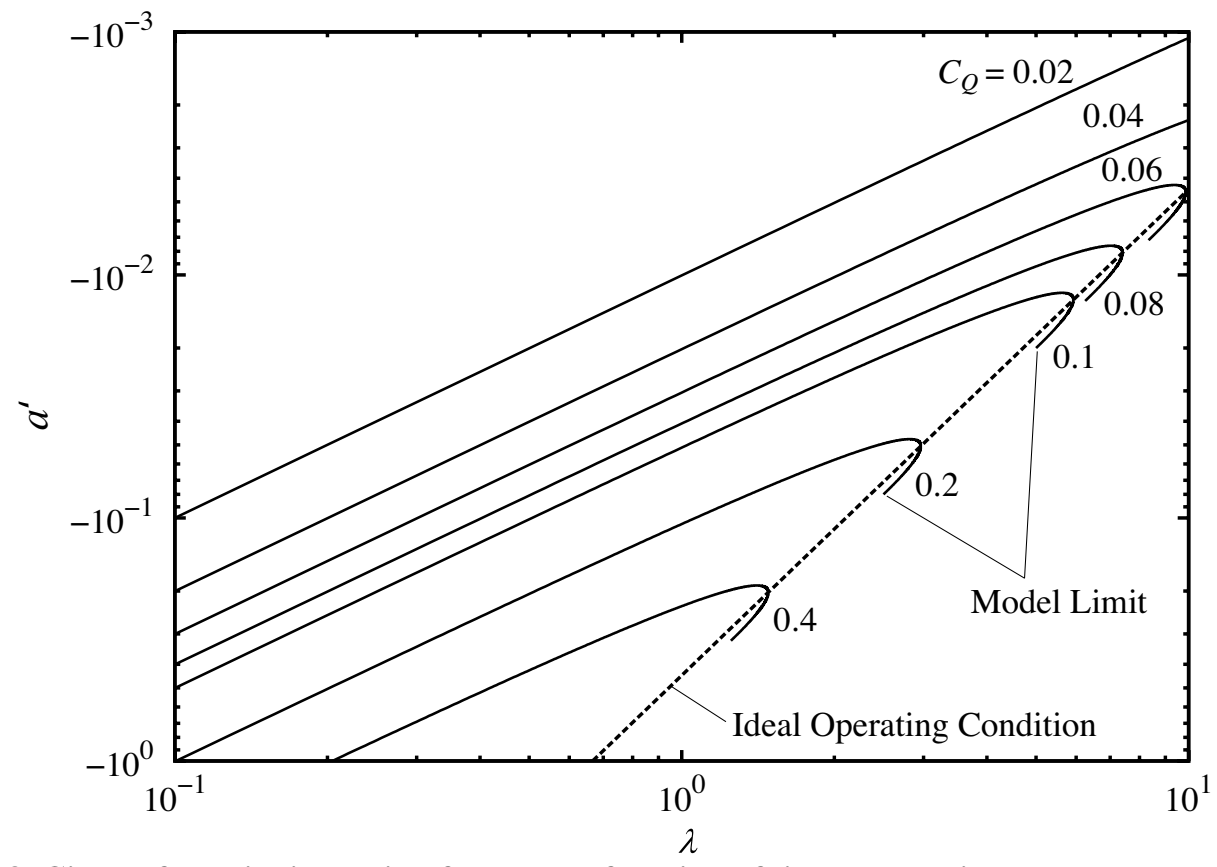

Figure 8. Circumferential induction factor as a function of tip-speed ratio and torque coefficient. 


\section{Conclusions}

A wind-turbine momentum theory has been presented which includes the effects of wake rotation and accounts for the radial pressure gradients in the slipstream. The momentum equation including angular momentum effects and Bernoulli's equation are directly applied to the case of rotational flow in a streamtube to evaluate the power and thrust coefficients of a turbine as a function of operating condition. This model is significant because it is the analog of the classical momentum theory, but includes the effects of wake rotation. Additionally, it does not suffer from the unrealistic approximations made by traditional momentum theory models which have attempted to account for slipstream rotation.

Care has been taken to present the results of the dependent variables of the model, which are the thrust coefficient, power coefficient, axial induction factor, and circumferential induction factor, in terms of two independent variables, which are the tip-speed ratio and torque coefficient. This is significant because it differs from the mainstream method for presenting similar results, which is to treat the axial induction factor as if it were an independent variable. Because the axial induction factor, just like the power coefficient and thrust coefficient, is dependent on the design and operating conditions of the turbine, it should not be viewed as an independent variable. Thus, Eqs. (55) and (56) are simply relations among the three dependent variables, $a, C_{T}$, and $C_{P}$ and the independent variable, $\lambda$. A significant contribution of this work has been to show the dependence of the axial induction factor on the two independent variables, the tip-speed ratio and torque coefficient. This relationship is given in Eqs. (71) and (72). Once this is known, the additional dependent variables including the thrust coefficient, power coefficient, and circumferential induction factor can be evaluated as functions of the two independent variables from Eqs. (55), (56), and (77). Figures showing plots for each of these relationships have been presented.

Results predicted from this wind-turbine model support previous claims that accounting for rotation within the slipstream does not affect the upper limit on wind-turbine efficiency known as the Lanchester-Betz-Joukowski limit. The model presented here predicts that the ideal efficiency of a wind turbine is $C_{P}=16 / 27$, even when rotational effects are included. This model predicts that the effects of slipstream rotation on wind-turbine performance are manifest entirely as an increase in the thrust force acting on the turbine disk for a given power coefficient. Additionally, it has been shown that neglecting the effects of slipstream rotation is not a conservative approach to thrust estimation. This is consistent with what is observed from propeller momentum theory, which predicts that slipstream rotation results in a decrease in thrust for a given power coefficient. Slipstream rotation should always be accounted for in thrust predictions of propellers or turbines.

The model predicts that maximum efficiency will always be achieved when the tip-speed ratio is related to the torque coefficient according to Eq. (61). Results for each of the dependent variables at ideal operating conditions are shown on the appropriate figures.

A final word of caution may be in order regarding the use of this or any other wind-turbine momentum theory. Results presented here have shown that, even when the effects of slipstream rotation are included, the approximations used in the development of wind-turbine momentum theory beak down completely in the limit as the axial induction factor approaches $a=1 / 2$. In this limit, wind-turbine momentum theory predicts a zero axialvelocity component and an infinite cross-section for the ultimate slipstream. The foundational hypothesis of a streamtube, which encloses the complete turbine disk as shown in Figs. 1 and 3, begins to break down at axial induction factors significantly below the absolute upper limit of $a=1 / 2$. In fact, this theory is strictly valid only in the limit as the axial induction factor approaches zero. Nevertheless, momentum theory including the effects of slipstream rotation provides some valuable insight into wind-turbine performance.

\section{References}

${ }^{1}$ Betz, A., "Das Maximum der theoretisch möglichen Ausnützung des Windes durch Windmotoren," Zeitschrift für das gesamte Turbinenwesen, Vol. 26, 1920, pp. 307-309.

${ }^{2}$ Lanchester, F. W., "A contribution to the theory of propulsion and the screw propeller," Transactions of the Institution of Naval Architects, Vol. 57, 1915, pp. 98-116.

${ }^{3}$ Bergey, K. H., "The Lanchester-Betz limit," Journal of Energy, Vol. 3, 1979, pp. 382-384.

${ }^{4}$ Joukowski, N. E., "Windmill of the NEJ type," Transactions of the Central Institute for Aero-Hydrodynamics of Moscow, 1920.

${ }^{5}$ Kuik, G. A. M., "The Lanchester-Betz-Joukowsky Limit,” Wind Energy, Vol. 10, 2007, pp. 289-291.

${ }^{6}$ Glauert, H., "Airscrew Theory," Aerodynamic Theory, Vol. 4, Durand, W. F. (Ed). Julius Springer, Berlin, 1935, p. 181. 
${ }^{7}$ Gorban, A. N., Gorlov, A. M., and Silantyev, V. M., "Limits of the Turbine Efficiency for Free Fluid Flow," Journal of Energy Resources Technology, Vol. 123, 2001, pp. 311-317.

${ }^{8}$ Cuerva, A., and Sanz-Andrés, A., “The extended Betz-Lanchester limit," Renewable Energy, Vol. 30, 2005, pp. 783-794.

${ }^{9}$ Jamieson, P., "Generalized Limits for Energy Extraction in a Linear Constant Velocity Flow Field," Wind Energy, Vol. 11, 2008, pp. 445-457.

${ }^{10}$ Okulov, V. L., and Sørensen, J. N., "Refined Betz Limit for Rotors with a Finite Number of Blades," Wind Energy, Vol. 11, 2008, pp. 415-426.

${ }^{11}$ Goldstein, S., "On the Vortex Theory of Screw Propellers," Proceedings of the Royal Society of London, Series $A$, Vol. 123, 1929, pp. 440-465.

${ }^{12}$ Huleihil, M., "Maximum windmill efficiency in finite time," Journal of Applied Physics, Vol. 105,2009 pp. 104908-1-104908-4.

${ }^{13}$ Werle, M. J., "Wind Turbine Wall-Blockage Performance Corrections," Journal of Propulsion and Power, Vol. 26, 2010, pp. 1317-1321.

${ }^{14}$ Joukowski, N. E., Travaux du Bureau des Calculs et Essais Aéronautiques de l'Ecole Superièure Technique de Moscou, 1918.

${ }^{15}$ Glauert, H., "The General Momentum Theory," Aerodynamic Theory Vol. 4, Durand, W. F. (Ed). Julius Springer, Berlin, 1935, pp. 191-201.

${ }^{16}$ Sharpe, D. J., “A General Momentum Theory Applied to an Energy-extracting Actuator Disc," Wind Energy, Vol. 7, 2004, pp. 177-188.

${ }^{17}$ de Vries, O., "Fluid Dynamic Aspects of Wind Energy Conversion," AGARD-AG-243, 1979.

${ }^{18}$ Wilson, R. E., Lissaman, P. B. S., "Applied Aerodynamics of Wind Power Machines," National Science Foundation Report NSF-RA-N-74-113, Oregon State University, 1974.

${ }^{19}$ Phillips, W. F., "Propeller Momentum Theory with Slipstream Rotation," Journal of Aircraft, Vol. 39, 2001, pp. 184-187.

${ }^{20}$ Hunt, V. D., “Applied Aerodynamics,” Windpower, Van Nostrand Reinhold Company, New York, 1981, pp. 100-130.

${ }^{21}$ Burton, T., Sharpe, D., Jenkins, N., and Bossanyi, E., "The Actuator Disc Concept," Wind Energy Handbook, Wiley, Chichester England, 2001, pp. 42-46.

${ }^{22}$ McCormick, B. W., "Momentum Theory," Aerodynamics, Aeronautics, and Flight Mechanics, 2nd ed. Wiley, New York, 1995, pp. 291-297.

${ }^{23}$ Phillips, W. F., "Propeller Momentum Theory," Mechanics of Flight, 2nd ed. Wiley, New Jersey, 2010 , pp. 202-216.

${ }^{24}$ Manwell, J. F., McGowan, J. G., and Rogers, A. L., "One-Dimensional Momentum Theory and the Betz Limit," Wind Energy Explained: Theory, Design and Application, Wiley, Chichester England, 2002, pp. 84-88.

${ }^{25}$ Burton, T., Sharpe, D., Jenkins, N., and Bossanyi, E., "Rotor Disc Theory," Wind Energy Handbook, Wiley, Chichester England, 2001, pp. 46-51. 